\title{
Optimization and comparison of different modes of preparative chromatography*
}

\author{
A. Seidel-Morgenstern
}

Otto-von-Guericke-Universität Magdeburg, Institut für Verfahrenstechnik and Max-Planck-Institut Dynamik Komplexer Tecknischer

System, Universitätsplatz, 2, D-39106 Magdeburg, Germany

Four different modes of preparative chromatography are presented, modeled and compared: classical elution, recycling chromatography, simulated moving bed chromatography and annular chromatography. To describe these modes a uniform model based on the partial differential equations derived from the column mass balances is used. The particularities of each mode are incorporated by specification of proper boundary conditions. Based on experimental data for the separation of two isomers of a steroid compound a quantitative comparison is performed with respect to different objective functions.

\section{List of symbols}

A $\quad\left[\mathrm{m}^{2}\right] \quad$ Cross section area.

$b \quad\left[\mathrm{~m}^{3} / \mathrm{kg}\right] \quad$ Adsorption isotherm parameter, equation (2).

c $\left[\mathrm{kg} / \mathrm{m}^{3}\right] \quad$ Concentration in the mobile phase.

d [m] Column diameter.

$d_{\mathrm{I}} \quad[\mathrm{m}] \quad$ Inner diameter of the annular chromatograph.

$d_{\mathrm{P}} \quad[\mathrm{m}] \quad$ Particle diameter.

* Abridged translation of a paper published recently in German by C. Heuer, H. Kniep, T. Falk and A. Seidel-Morgenstern (Chemie Ingenieur Technik 69, 1997, 1535-1546). 
$D_{\text {ap }} \quad\left[\mathrm{m}^{2} / \mathrm{s}\right] \quad$ Apparent dispersion coefficient.

$D_{\mathrm{m}} \quad\left[\mathrm{m}^{2} / \mathrm{s}\right] \quad$ Molecular diffusion coefficient.

$D_{\text {tan }}\left[\mathrm{m}^{2} / \mathrm{s}\right] \quad$ Tangential dispersion coefficient.

$E C \quad\left[\mathrm{~m}^{3} / \mathrm{kg}\right] \quad$ Specific eluent consumption.

$F \quad[-] \quad$ Phase ratio, $F=(1-\varepsilon) / \varepsilon$.

$K \quad[-] \quad$ Number of columns (SMB).

$L \quad[\mathrm{~m}] \quad$ Column length.

$h$ [m] Reduced height of a theoretical plate, $h=L /\left(N d_{\mathrm{p}}\right)$.

$m \quad[\mathrm{~kg}] \quad$ Mass

$M \quad[-] \quad$ Number of components.

$N \quad$ [-] Number of theoretical plates.

$n_{\mathrm{Zyk}} \quad[-] \quad$ Number of recycling steps (recycling chromatography).

$P R \quad\left[\mathrm{~kg} /\left(\mathrm{s} \mathrm{m}^{2}\right)\right]$ Productivity or production rate.

Pur [\%] Product purity.

$q \quad\left[\mathrm{~kg} / \mathrm{m}^{3}\right] \quad$ Loading of the stationary phase.

$q_{\mathrm{S}} \quad\left[\mathrm{kg} / \mathrm{m}^{3}\right] \quad$ Saturation loading of the stationary phase.

REC [-] Recovery.

$t \quad[\mathrm{~s}] \quad$ Time.

$t_{0} \quad[\mathrm{~s}] \quad$ Dead time.

$t_{\mathrm{R}} \quad[\mathrm{s}] \quad$ Retention time.

$t_{\mathrm{Zyk}}[\mathrm{s}] \quad$ Cycle time (time between two injections for discontinuous operating modes).

$T_{\mathrm{S}} \quad$ [s] $\quad$ Switching time (SMB).

$u \quad[\mathrm{~m} / \mathrm{s}] \quad$ Linear velocity, $u=\frac{\dot{V}}{E A}$.

$V \quad\left[\mathrm{~m}^{3}\right] \quad$ Volume.

$V_{\text {stat }}\left[\mathrm{m}^{3}\right] \quad$ Volume of the stationary phase,

$V_{\text {stat }}=(1-\varepsilon) \cdot A \cdot L$.

$\dot{V} \quad\left[\mathrm{~m}^{3} / \mathrm{s}\right] \quad$ Flow rate.

$w_{1 / 2}[\mathrm{~s}] \quad$ Peak width at half height.

$z \quad[\mathrm{~m}] \quad$ Coordinate in axial direction.

$e \quad[-] \quad$ Porosity, $\varepsilon=\left(V-V_{\text {stat }}\right) / V$.

$v \quad[-] \quad$ Reduced linear velocity, $v=\left(\begin{array}{ll}u & d_{\mathrm{p}}\end{array}\right) / D_{\mathrm{m}}$.

$j \quad$ [rad] Angular coordinate of the annular appara-

$\omega \quad[\mathrm{rad} / \mathrm{min}]$ Rotation speed of the annular apparatus.

Upper indices

$k$

1,2

I, II, III, IV

Column number index.

First and second center, equation (2).

Number of zone (SMB).

Transformed coordinate.

Lower indices

a

e

F

$i$

inj

S

$\mathrm{s}$

Zyk

Begin of the collection.

End of the collection.

Feed.

Component $i$.

Injected.

Solvent.

Switching.

Cycle. substantial progress achieved in producing selective and stable stationary phases [1]. Further, from a process engineering point of view, there is now a better understanding of the development of concentration profiles in chromatographic columns under overloaded conditions available [2]. This includes in particular the quantitative description of displacement and tag-along effects caused by competitive adsorption. Besides, a series of alternative continuous and discontinuous chromatographic modes has been developed to improve yields and production rates [3]. However, the studies performed hitherto are usually focused on the design and optimization of one of the available modes. In contrast, the objective of this paper is to compare the performance of four different modes of preparative chromatography on the basis of a uniform theoretical model.

\section{Modes of operation in preparative chromatography}

The following concepts will be discussed and compared:

1) Classical isocratic elution chromatography.

2) Recycling chromatography.

3) Simulated moving bed chromatography (SMB).

4) Annular chromatography (AC).

The chemical engineering approach used for the description and optimization of each of these concepts might be adapted to other modes not considered here as e.g. displacement or two-way chromatography.

The four mentioned modes are illustrated schematically in figure 1. Since the principle is well-known from analytical chromatography no further explanation is required regarding classical elution chromatography.

Recycling chromatography is characterized by the fact that the sample to be separated is transported several times through the column. Thus, a longer column is simulated leading to an improvement of separation until a situation is reached when the sample is spread over the whole column and subsequent cycles begin to overlap [4]. To postpone this undesired effect and to reach higher yields there exists the possibility to take in each cycle sufficiently purified fractions out of the recycle loop (peak shaving).

The simulated moving bed chromatography uses a series connection of several columns in combination with a cyclic variation of feed and collecting positions. This mimics a counter-current between mobile and stationary phases. The concept is already a long time successfully in use to perform relative simple separations (e.g. isomers of the xylenes, glucose-fructose). Recently, intensive investigations have been carried out to apply it for more difficult separations. The SMB process is thoroughly described e.g. in $[5,6]$.

The process of annular chromatography uses a stationary phase fixed in the annular space between two concentrical cylinders. Through the fixed bed the mobile phase is transported from top to bottom. In a certain angular region the mixture to be separated is continuously dosed on top. The fixed-bed rotates slowly. Thus, a cross-flow between mobile and stationary phases is established. The components of the 


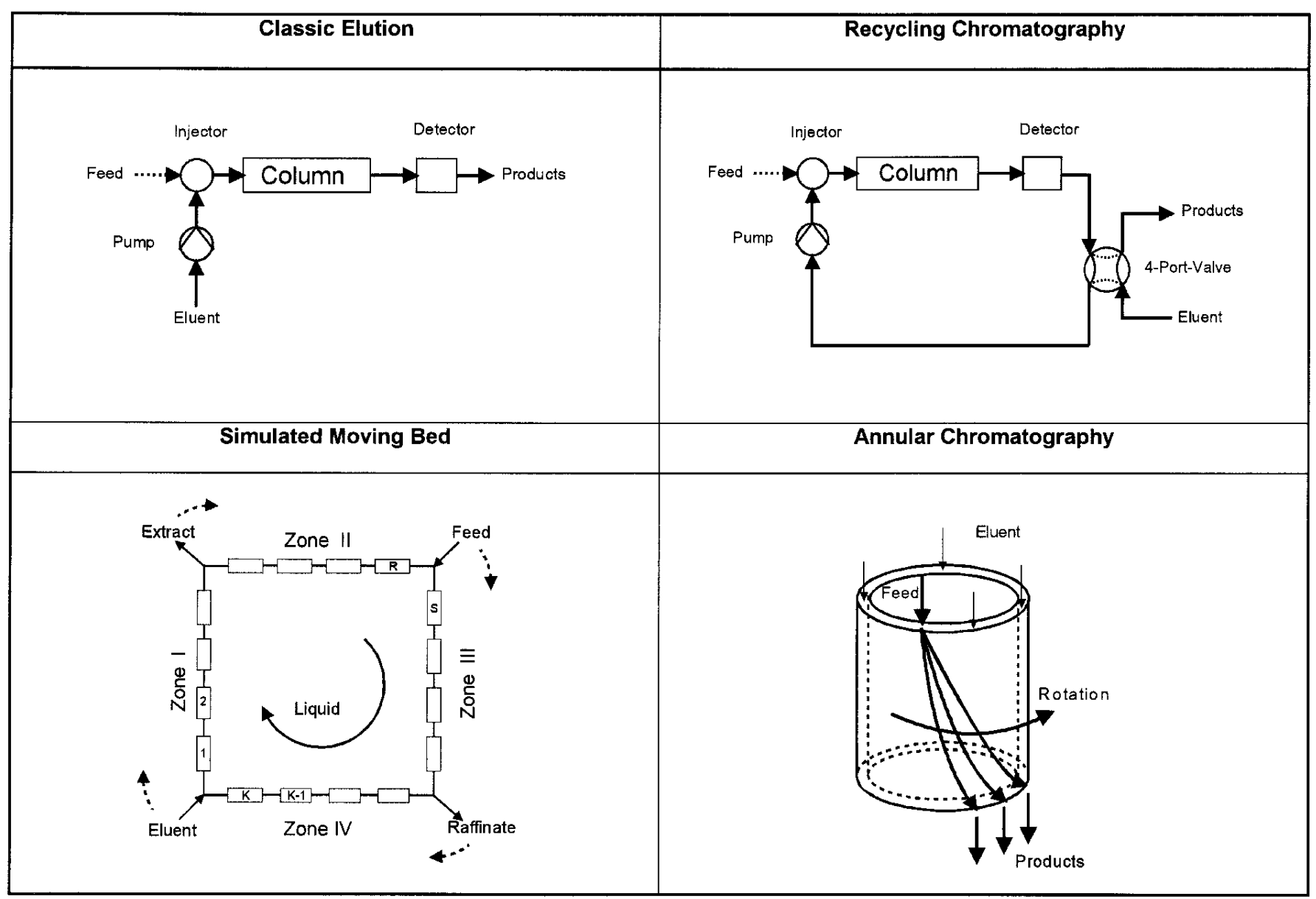

Figure 1. Schemes of the four investigated chromatographic modes.

feed separate from each other in helical bands on their way downwards [7].

An obvious and essential difference between the four modes discussed above is the fact that elution and recycling operate discontinuously whereas SMB and AC offer continuous operation.

In the following all four modes will be compared on the basis of a uniform mathematical model with respect to different objective functions. To determine realistic parameters and to verify the model, preliminary experiments were carried out using elution and recycling for the separation of two isomers of a steroid compound on silica.

\section{Modeling preparative chromatography}

To simplify the quantification of the chromatographic separation process the following assumptions can be used:

- since the columns are usually well packed, radial concentration gradients can be neglected,

- there are relative small particles applied, thus intraparticle concentration gradients are also negligible,
- the two phases can be considered as quasi-homogeneous, i. e. adsorption equilibria are permanently established throughout the column,

- due to relative low thermal effects, isothermal conditions can be assumed.

Then, to describe the local and temporal course of the concentration profiles of a component $i$ in a chromatographic column, the classical equilibrium dispersion model can be used [2]:

$\frac{\partial c_{i}}{\partial t}+F \frac{\partial q_{i}(\vec{c})}{\partial t}+u \frac{\partial c_{l}}{\partial z}=D_{a p} \frac{\partial^{2} c_{l_{1}}}{\partial z^{2}} \quad i=1, M$.

In equation (1) $c$ is the concentration in the mobile phase and $q$ is the loading of the stationary phase assumed to be in equilibrium with the mobile phase concentration, $F$ is the phase ratio that results from the porosity and $u$ is the linear velocity of the mobile phase. The task of the apparent dispersion coefficient $D_{\text {ap }}$ is to describe all kinetic effects that cause band broadening. The model was applied successfully in the last years for the description of the chromatographic separation of rather different feed mixtures [2].

To calculate chromatographic separations the knowledge of the adsorption isotherm $q(\vec{c})$, quantifying the distribution of a component between the two phases, is an essential supposition. To describe the equilibrium loading of a 
component $i$ on energetically heterogeneous surfaces the following extension of the Langmuir equation assuming two types of adsorption sites has been proven to be a reliable and versatile tool $[2,8]$ :

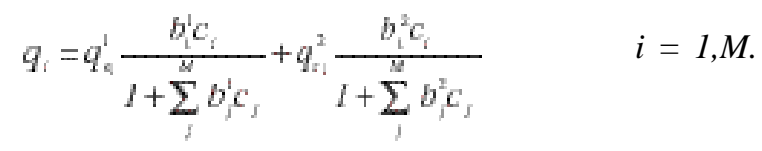

This equation has four free parameters for each component. These are the saturation capacities of the two sites, $q_{\mathrm{s}}{ }^{1}$ and $q_{\mathrm{s}}^{2}$, and the constants $b^{l}$ and $b^{2}$.

For low values of $D_{\text {ap }}$ the well-known analogy between the dispersion model (Eq. 1) and a cell model [e.g. 2] holds, provided the following relation between the model parameters is fulfilled:

$$
D_{\nsim}=\frac{u \cdot L}{2 \cdot N}
$$

The number of cells or the number of theoretical plates, $N$, can be determined in a standard way by an evaluation of the statistical moments of experimental elution profiles obtained from very small sample sizes characteristic in analytical chromatography. If the profiles can be approximated by a Gaussian distribution the following well known estimation formula holds true:

$$
N=5.54\left(\frac{t_{\underline{z}}}{w_{x z}}\right)^{2}
$$

where $t_{\mathrm{R}}$ is the retention time and $w_{1 / 2}$ the width of the peak at half height. The classical work by van Deemter et al. [9] explained and quantified the fact that the number of theoretical plates $N$ is a function of the mobile phase velocity in the column, $u$. A rough estimation of the dependence $N(u)$ can be performed using the dimensionless Knox equation [2]:

$$
h-v^{y 3}+\frac{2}{v}+\frac{v}{10}
$$

In this equation are $h=L /\left(N d_{\mathrm{P}}\right)$ the reduced plate height, $v=\left(\begin{array}{ll}u & d_{\mathrm{P}}\end{array}\right) / D_{\mathrm{m}}$ the reduced velocity, $L$ the column length, $d_{\mathrm{P}}$ the diameter of the particles and $D_{\mathrm{m}}$ the molecular diffusion coefficient of a component in the mobile phase.

Equation (1) can be used to describe separations in conventional fixed beds as applied in elution, recycling chromatography and in the SMB process provided the phase ratio $F$, the adsorption isotherm $q(\vec{c})$ and the apparent dispersion coefficient $D_{\text {ap }}$ are known. An equivalent description of the concentration profiles in an annular chromatograph has to consider the angle $\varphi$ as an additional spatial coordinate. The mass balance of the AC corresponding to the assumptions given above is:

$$
\frac{\partial c_{t}}{\partial t}+F \frac{\partial q_{i}(\vec{c})}{\partial t}+u \frac{\partial c_{t}}{\partial z}+\omega \frac{\partial c_{t}}{\partial \varphi}+\omega F \frac{\partial q_{i}(\vec{c})}{\partial \varphi}=D_{\varphi} \frac{\partial^{2} c_{t}}{\partial z^{2}}+D_{i=} \frac{\partial^{2} c_{t}}{\partial \varphi^{2}}
$$

Equation (6) contains as additional parameters the rotation speed $\omega$ and the tangential dispersion coefficient $D_{\tan }$. With the further assumptions that the AC process should have reached its steady state $(\partial / \partial t=0)$ and that the tangential dispersion is negligible $\left(D_{\tan }=0\right)$ a transformation, $t^{*}=\varphi /$ $\omega$, simplifies equation (6) [10]:

$$
\frac{\partial c_{i}}{\partial t^{*}}+F \frac{\partial q_{i}(\vec{c})}{\partial t^{*}}+u \frac{\partial c_{i}}{\partial z}=D=\frac{\partial^{2} c_{i}}{\partial z^{2}}
$$

Obviously equation (7) corresponds to equation (1). Thus, the temporal concentration profile at a certain position of a column characterizing the elution process corresponds to a profile over the angular coordinate in annular chromatography [11].

Consequently, equation (1) can be used uniformly to compare all processes considered here if the underlying assumptions are acceptable. To incorporate the specific character of each process, a proper formulation of the corresponding initial and boundary conditions is necessary. Usually not preloaded adsorbents are applied leading to the following initial conditions:

$$
c_{i}(z, t=0)=0 \quad \text { rsp. } \quad c_{i}\left(z, t^{*}=0\right)=0
$$

For the two discontinuous processes (elution and recycling) holds at the columns inlet:

$$
\begin{aligned}
& 0 \leq t<t_{\text {inj }}: c_{i}(z=0, t)=c_{\mathrm{F}, i} \quad \text { (injection) } \\
& t \geq t_{\mathrm{inj}}: \quad c_{i}(z=0, t)=0 \quad \text { (elution) } \\
& c_{i}(z=0, t)=c_{i}(z=L, t) \quad \text { (recycling). }
\end{aligned}
$$

Since in the continuous SMB process the columns are connected in series, for all columns, with the exception of the positions where feed or solvent is introduced, hold the following boundary conditions:

$$
c_{i}^{k}(z=0, t)=c_{i}^{k-1}(z=L, t) \quad k=2, K .
$$

In the boundary conditions for the first column in zone I the mixing of the effluent of the last column of zone IV with fresh solvent has to be considered (compare Fig. 1):

$$
c^{\prime}(z=0, t)=c^{k}(z=L, t) \frac{V_{t w}}{V_{1}}
$$

Further, for the first column in zone III after the feed port holds (compare again Fig. 1):

$$
q^{5}(z=0, t)=\frac{1}{V_{n}}\left(c^{n}(z=L, t) \cdot V_{n}+c_{n}, V_{r}\right) \text {. }
$$

The specification of the fluid phase velocities and the switching times required to guarantee a successful separation with the SMB process will not be discussed in detail here. The determination of appropriate values can be performed using the equilibrium theory assuming an infinite number of theoretical plates $[11,12]$. The range of applicability of this concept for finite plate numbers was analyzed by numerical simulations. A detailed description is given in [13].

The boundary conditions for annular chromatography are in analogy to the elution process:

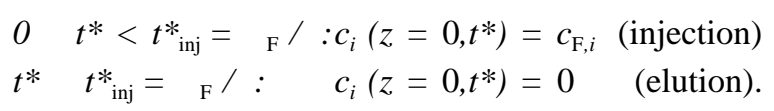

To avoid hydrodynamic perturbations around the feed position and to assure a constant velocity in the whole apparatus, the following relation has to be fulfilled by the 
volumetric flowrates of the feed solution, $\dot{V}_{\mathrm{F}}$, and the solvent, $\dot{V}_{\mathrm{S}}$ :

$$
\dot{V}_{p}=\frac{\varphi_{F}}{2 \pi-\varphi_{p}} \dot{V}_{s}
$$

Usually the adsorption isotherms, $q_{i}(\vec{c})$, are nonlinear (e.g. Eq. (2)). Thus, there are no analytical solutions available to solve equations (1) and (7) and numerical methods must be used. Favorably the Godunov method can be applied exploiting quantitatively the knowledge about numerical dispersion effects that are caused by usage of finite difference approximations. The method allows the application of rather coarse grids leading to fast calculations [14]. About the adaptation to simulate multi-column counter-current processes is reported in detail in [13]. The application of the described model and the mentioned numerical solution allows the simulation of all four chromatographic modes on a personal computer within a few minutes.

\section{Parameters and model verification}

There are the following free parameters of the model based on equation (1): the phase ratio $F$, the thermodynamic properties of the system expressed by the adsorption isotherms $q(\vec{c})$ and the axial dispersion coefficient $D_{\text {ap }}$ or the number of separation plates $N$, respectively, that describe process kinetics.

For a comparative study parameters determined for the separation of two isomers ( $\alpha$ - and $\beta$-isomer, respectively) of a steroid compound with silica as the stationary phase (particle diameter $10 \mu \mathrm{m}$, EKA Nobel) and a mixture of $n$ hexane and methyl-tert.-butyl-ether (70:30) as the mobile phase were used. The applied semi-preparative column had a length of $25 \mathrm{~cm}$ and an internal diameter of $0.8 \mathrm{~cm}$. The volume of this column served as the base for the comparison presented below. A more detailed description of the chromatographic system and the experiments carried out is given elsewhere $[8,15]$.

From the retention time of a solvent impulse the phase ratio was estimated to be $F=0.5$. The adsorption isotherms of both isomers were measured using the Elution by Characteristic Point method (ECP) [16,17]. They could be successfully correlated with equation (2). In table I the obtained parameters are given. The number of theoretical plates of the applied column was determined using equation (4) to be $N=6500$ for a linear velocity $u=0.2 \mathrm{~cm} / \mathrm{s}$. From this value a diffusion coefficient of $D_{\mathrm{m}}=1.3 \cdot 10^{-9} \mathrm{~m}^{2} / \mathrm{s}$ can be estimated with equation (5) allowing to calculate for an arbitrary linear velocity $u$ the actual number of theoretical plates $N(u)$.

In order to evaluate the applicability of the described model, equation (1), and the determined parameters (Tab. I) a comparison between experimental and simulated elution profiles was performed. The separation of the two isomers was analyzed as well with the classical elution process as with recycling chromatography. In figures 2 and 3 typical results are shown for the two different regimes. Considering the simplicity of the applied model the accuracy between experimental data and predictions is satisfactorily. Even for the more complex recycling process with peak shaving
Table I. Parameters for the separation of $\alpha$ - and $\beta$-isomers.

\begin{tabular}{lcccc}
\hline & \multicolumn{5}{c}{ Adsorption isotherm, equation (2) } \\
\hline$L[\mathrm{~cm}]$ & 25 & & $\alpha$-isomer & $\beta$-isomer \\
$d[\mathrm{~cm}]$ & 0.8 & $q_{\mathrm{s}}^{1}[\mathrm{~g} / \mathrm{l}]$ & 453.0 & 376.8 \\
$F$ & 0.50 & $b^{1}[\mathrm{l} / \mathrm{g}]$ & 0.010 & 0.017 \\
$V_{\text {stat }}[\mathrm{ml}]$ & 12.57 & $q_{\mathrm{s}}^{2}[\mathrm{~g} / \mathrm{l}]$ & 2.470 & 3.248 \\
$\dot{V}[\mathrm{ml} / \mathrm{min}]$ & 4 & $b^{2}[\mathrm{l} / \mathrm{g}]$ & 0.377 & 0.492 \\
$N$ & 6500 & & & \\
$D_{\mathrm{P}}[\mu \mathrm{m}]$ & 10 & & & \\
$D_{\mathrm{m}}\left[\mathrm{m}^{2} / \mathrm{s}\right]$ & $1.3 \cdot 10^{-9}$ & & & \\
\hline
\end{tabular}

(Fig. 3) a relative good agreement can be noticed. In the illustrated case there is a rather satisfying separation of the two components already after the first cycle. The second cycle is sufficient to achieve an almost complete separation. Also for other experimental conditions (varied were the amount injected, the composition of the feed mixture, the volumetric flowrate and the column dimensions [8]) the quality of the agreement between experimental and theoretical chromatogram as shown in figures 2 and 3 could be confirmed.

The obtained results to separate the two isomers demonstrate the applicability of the described dispersion model in combination with the experimentally determined adsorption isotherms and the boundary conditions formulated for the quantitative description of different types of chromatographic processes. Similar results, that are not presented here, were obtained for another experimental example in verifying the model for predicting the performance of the SMB process [13].

\section{Optimization and comparison of methods}

In order to compare different modes it should be emphasized that for a given chromatographic system of dissolved components, stationary phase and mobile phase the adsorption isotherms as thermodynamic quantities have to be used uniformly. Thus, to describe all four concepts equation (2) and the parameters given in table I have been used. Concerning the composition of the binary feed only 1:1 mixtures were considered and a maximum concentration of $200 \mathrm{~g} / \mathrm{L}$ for each component was set as an upper limit. In order to perform a quantitative comparison a few more assumptions were made. At first, in the calculations for all processes the same small amount of stationary phase used in the experimental lab-scale investigations was applied $\left(V_{\text {stat }}=12.57 \mathrm{~mL}\right)$. For the SMB process this amount was distributed evenly in 8 columns with the diameter of the column used in the elution and recycling study $(d=0.8 \mathrm{~cm})$. The annular chromatograph was considered to possess the same bed height as the column used for elution $(25 \mathrm{~cm})$. A diameter of the inner cylinder of $d_{\mathrm{I}}=3 \mathrm{~cm}$ was chosen. The phase ratio $F$ was uniformly assumed to be 0.50 . Further, concerning the relation between linear velocity $u$ or flowrate $\dot{V}$, rsp., and dispersion coefficient $D_{\text {ap }}$ and plate number $N$, 


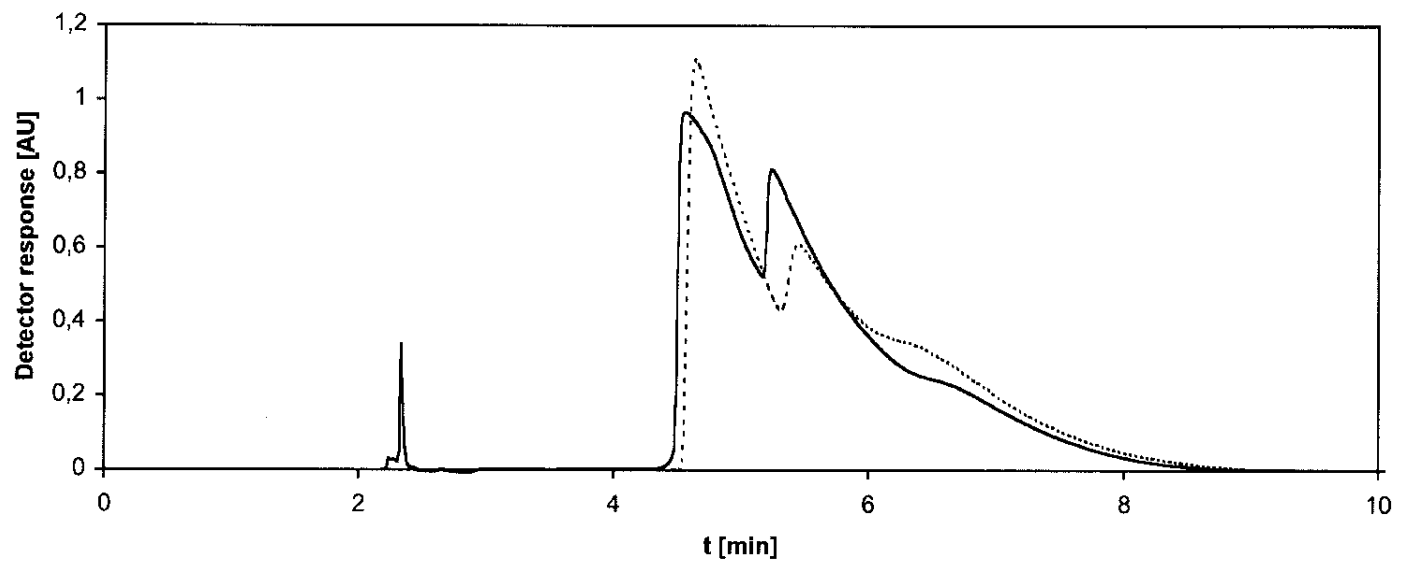

Figure 2. Elution mode. Experimental (solid) and calculated elution profile (dotted) for the separation of a 1:1-mixture $\left(m_{\mathrm{inj}}=120 \mathrm{mg}\right)$ of $\alpha$ - and $\beta$-isomers.

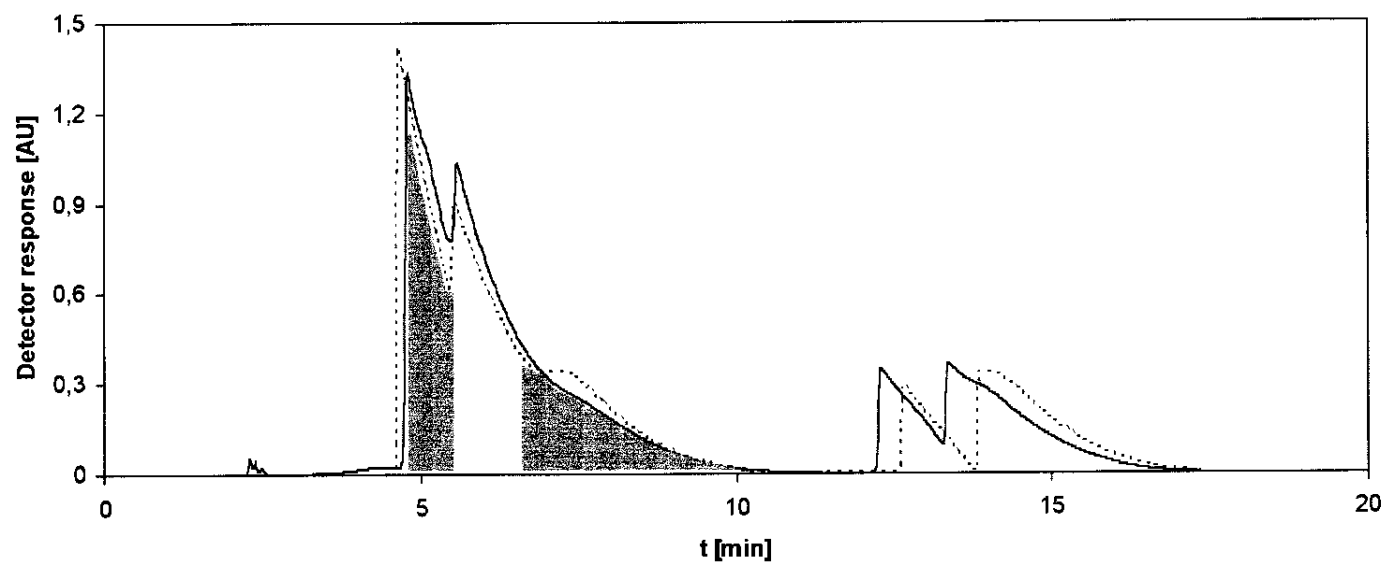

Figure 3. Recycling with peak-shaving for two cycles. Experimental (solid) and calculated elution profile (dotted) for the separation of a 1:1-mixture $\left(m_{\mathrm{inj}}=200 \mathrm{mg}\right)$ of $\alpha$ - and $\beta$-isomers. The mixture between the shaded area is recycled.

rsp., uniformly the validity of equation (5) was assumed. The two last assumptions are rather optimistic with respect to the annular apparatus due to the fact that the packing technology has not yet reached the level available nowadays for standard HPLC columns. If the mentioned assumptions are valid the results that can be achieved with the annular apparatus correspond, due to the analogy between equations (1) and (7), to the results of the classical elution process. As additional constraints in all calculations, minimum purities for the product fractions were set to be Pur $=99 \%$ and minimum recovery yields were specified to be $R E C=0.95$. These two parameters, together with other quantities suitable to characterize the success of a separation, are defined in table II. To evaluate the classical elution process, in the literature frequently the recovery yield $R E C$ and the production rate $P R$ (e.g. [18]) or a combination of these two [19] are applied as objective functions. Further, it might be important to consider the specific eluent consumption $E C$. In this work the three performance parameters $R E C, P R$ and $E C$, defined in table II, were calculated with the discussed mathematical model in a range of operating parameters specific for each mode. Table III summarizes the covered oper- ating parameters of all modes. The regions where a separation is possible and a further evaluation is reasonable are also given. The streams leaving the different units were only considered to be useful for production if the concentration was at least $0.5 \%$ of the feed concentration. Table III further describes specific peculiarities of the individual processes incorporated in the boundary conditions.

Below, a few examples of the obtained results will be reported. The main focus is set on the maximization of the production rates ( $\mathrm{PR} \rightarrow \mathrm{Max})$ and the minimization of the eluent consumptions (EC $\rightarrow$ Min). Only the performance parameters concerning the optimal production of the first eluting $\alpha$-isomer will be reported. However, despite of different absolute numbers, the main general tendencies hold as well for the production of the $\beta$-isomer.

Figure 4 shows selected results of simulating the elution process to demonstrate the influence of the injection volume $V_{\text {inj }}$ and the plate number $N$ (beeing according to Eq. (5) a measure of the flowrate) on the production rate $P R_{\alpha}$, the eluent consumption $E C_{\alpha}$ and the recovery yield $R E C_{\alpha}$. In all three parts of the figure the two operating points that lead 
Table II. Definition of performance parameters for the investigated chromatographic modes.

\begin{tabular}{|c|c|c|c|}
\hline & Recycling & $S M B$ & $A C$ \\
\hline Purity [\%] & \multicolumn{3}{|c|}{$P u r_{i}=100[\%] \frac{c_{i}}{\sum_{c_{i}}} ; \quad i=1, M$} \\
\hline \multirow[t]{2}{*}{ Produced mass [mg] } & $m_{i}=V_{s} \int_{4}^{r_{e j}} c_{L}(t) \mathrm{d} t$ & $m q_{i}^{S}=\dot{V}_{f} \cdot \int_{i v-s, g_{3}}^{w_{s}} c_{i}^{s}(t) \mathrm{d} t$ & 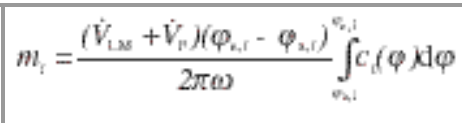 \\
\hline & & for tact $n$ and outlet $j$ & \\
\hline Productivity & $P R=\quad m_{t}$ & $P R^{\prime}=m_{i}^{\prime}$ & $\omega n_{i}$ \\
\hline$\left[\mathrm{mg} /\left(\mathrm{s} \mathrm{cm}^{2}\right)\right]$ & $R_{i, k}(1-\varepsilon) A$ & $P R_{r}^{\prime}=\overline{T^{u}(1-\varepsilon) M}$ & $P K_{i}=\overline{\left(\varphi_{z,}-\varphi_{z, i}\right)(1-\varepsilon) A}$ \\
\hline Recovery [ - ] & $R E C_{t}=\frac{m_{i}}{V}$ & $R E C_{r}^{\prime}=\frac{m}{r}$ & $R E C_{t}=\frac{\omega m m_{t}}{}$ \\
\hline & $V_{\mathrm{imi}} c_{\mathrm{i}}$ & $R E C_{i}=\overline{T_{\mathrm{s}} V_{\mathrm{z}} c_{\mathrm{z}, \mathrm{f}}}$ & $\overline{\left(\varphi_{e, t}-\varphi_{z, j}\right) \dot{V}_{y} c_{y, j}}$ \\
\hline $\begin{array}{l}\text { Specific eluent } \\
\text { consumption }[\mathrm{L} / \mathrm{g}]\end{array}$ & $E C_{t}=\frac{\dot{V}_{t} t_{\text {tsk }}}{m_{t}}$ & $E C_{i}=\frac{T^{x}\left(V_{L M}+\dot{V}_{F}\right)}{m_{l}}$ & $E C_{1}=\frac{\left.\left(\varphi_{n, 2}-\varphi_{21}\right) \dot{V}_{1}+\dot{V}_{V}\right)}{\omega m_{t}}$ \\
\hline
\end{tabular}

Table III. Free parameters and investigated areas for the four considered modes.

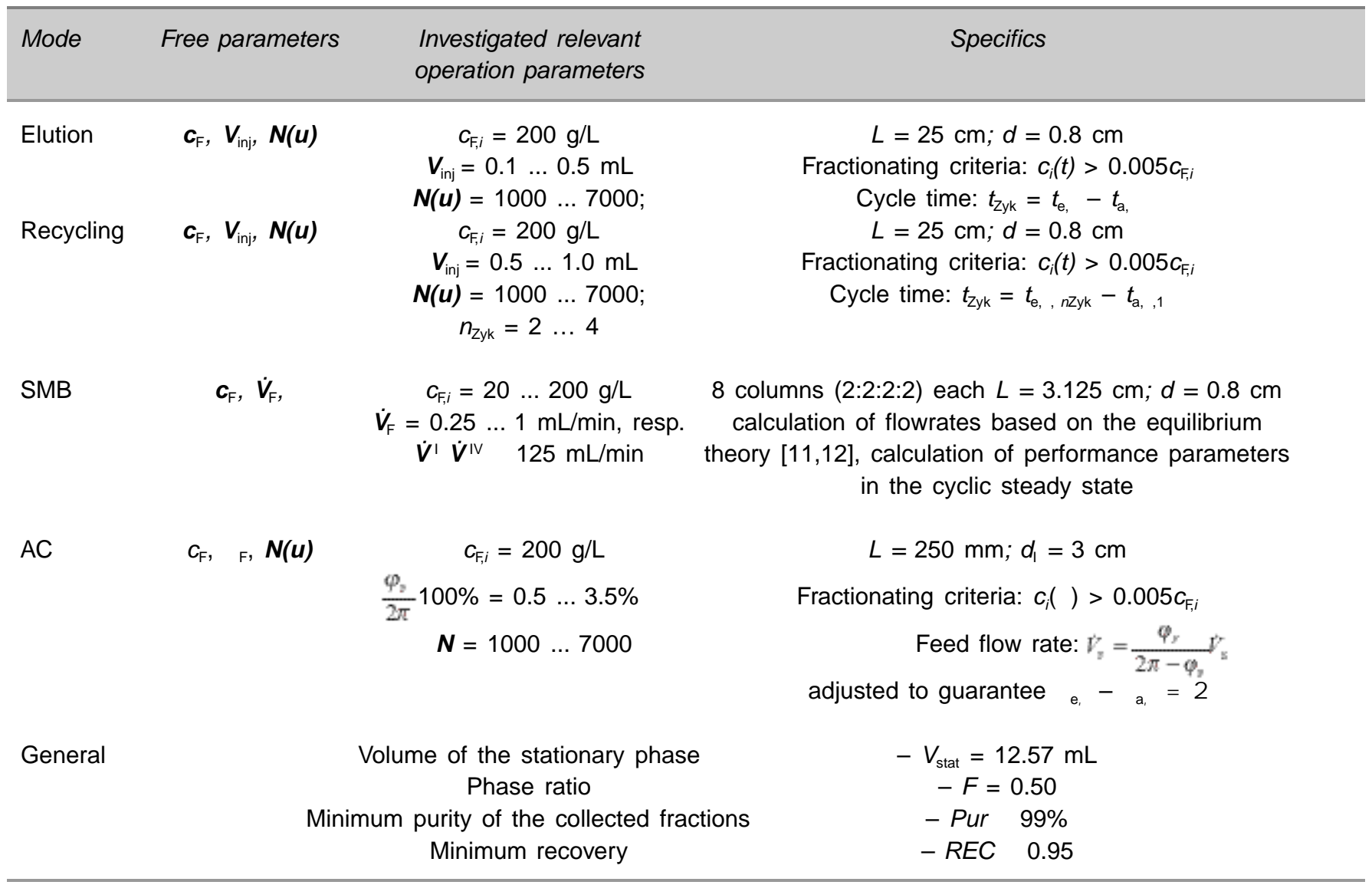

to a maximum production rate $\left(V_{\text {inj }}=0.3 \mathrm{~mL}, N=1000\right.$ or $\dot{V}_{\mathrm{S}}=50.4 \mathrm{~mL} / \mathrm{min}$ ) or a minimum eluent consumption $\left(V_{\text {inj }}=0.5 \mathrm{~mL}, N=7000\right.$ or $\dot{V}_{\mathrm{S}}=3 \mathrm{~mL} / \mathrm{min}$ ) and that fulfill the other constraints are marked. It is obvious that the two optima correspond to very different flowrates. For a concrete separation problem there is always a decision necessary which objective function is more important or if a combined function weighting different objectives might be preferable.
In figure 4 the obvious tendency can be recognized that increasing the injection volume or decreasing the plate number causes reduced recovery yields. With the classical elution process it is not possible for the given conditions to separate injection volumes larger $0.5 \mathrm{~mL}$ because the required recovery yields $(>0.95)$ can not be achieved above this value.

Figure 5 illustrates the essential effect of recycling chromatography on the recovery yields. Obviously, compared 

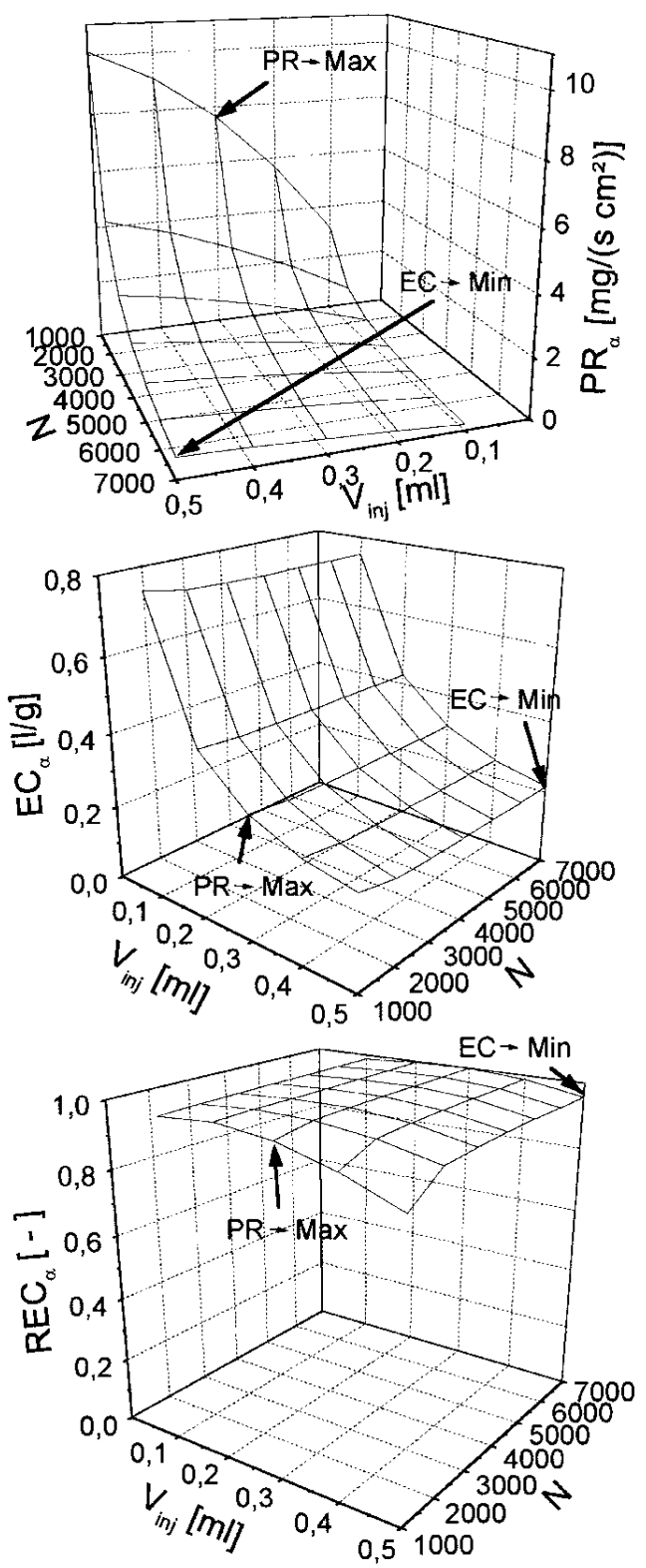

Figure 4. Performance parameters of the $\alpha$-isomer for the elu-

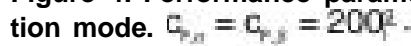

with elution chromatography larger injected amounts can be resolved (compare bottom of Fig. 4). The main reason is that there is not only one pass through the column available. However, the increased cycle time causes a reduction of the production rate using the recycling mode.

In figure 6 the influence of the feed flowrate and the feed concentration on the productivity of the SMB process is shown. Also for this process the optimum operating points corresponding to the maximum production rate and the minimum eluent consumption differ greatly. To improve both objective functions the feed concentration should be maximized. To select the feed flowrate the objective function is of essential importance. For industrially relevant separations often the production rate will be the essential parameter. To maximize this, the application of a large feed flowrate is advantageous. Concerning an increase of the two considered

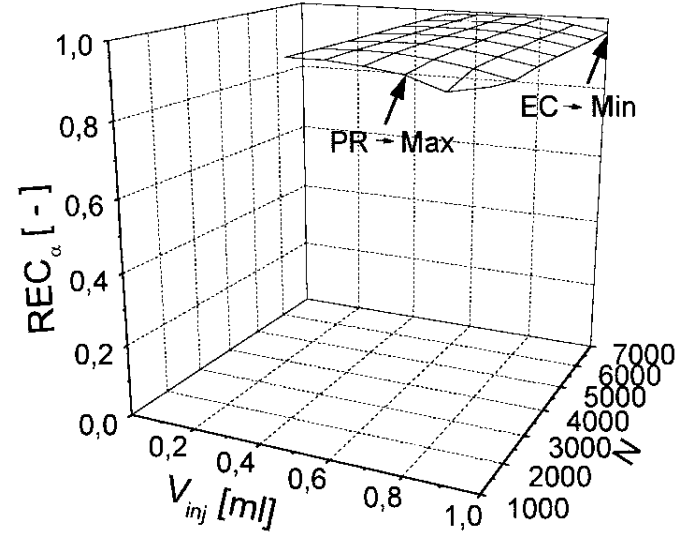

Figure 5. Recovery of the $\alpha$-isomer for the recycling mode with peak-shaving. $\bar{\sigma}_{s}=C_{y, j}=200^{k}, n_{\mathrm{zyk}}=2$ (resp. 3, if necessary for $R E C_{\alpha}>0.95$ ).

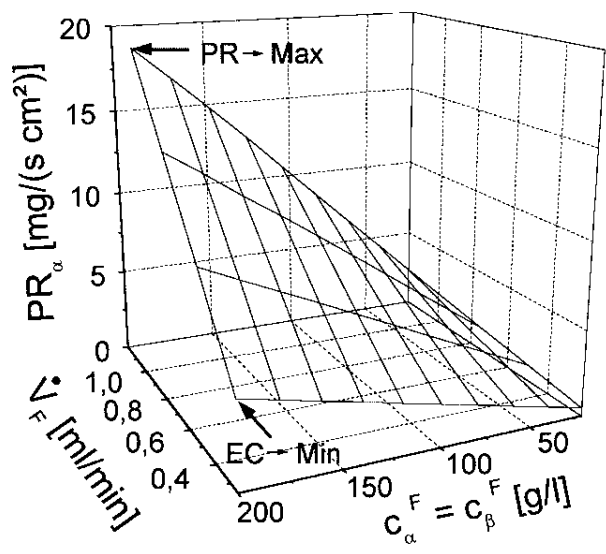

Figure 6. Productivity for the production of the $\alpha$-isomer with the SMB process as a function of feed concentration and feed flowrate.

free parameters, feed concentration and feed flowrate, it should be mentioned that the process becomes more sensitive with respect to perturbations and might even not be feasible under practical conditions where small flowrate fluctuations could not be avoided [12].

All determined operating points and the calculated performance parameters are given for the four processes in table IV. It is emphasized one more time that with the applied assumptions and neglecting round off errors the results of annular chromatography do not differ from the results of elution chromatography.

Comparing the performance parameters obtained with the objective function of the maximum production rate it can be recognized that the results for elution (or annular chromatography) and recycling chromatography are rather similar. The production rate of the SMB-process is considerably higher. This is also related to the fact that for the SMB process shorter columns were applied (the length of one zone was $1 / 4$ of the column length for the elution process) leading to reduced pressure drops and allowing for larger 
Table IV. Operating parameters, productivity and specific eluent consumption for the considered modes (production of the $\alpha$-isomer).

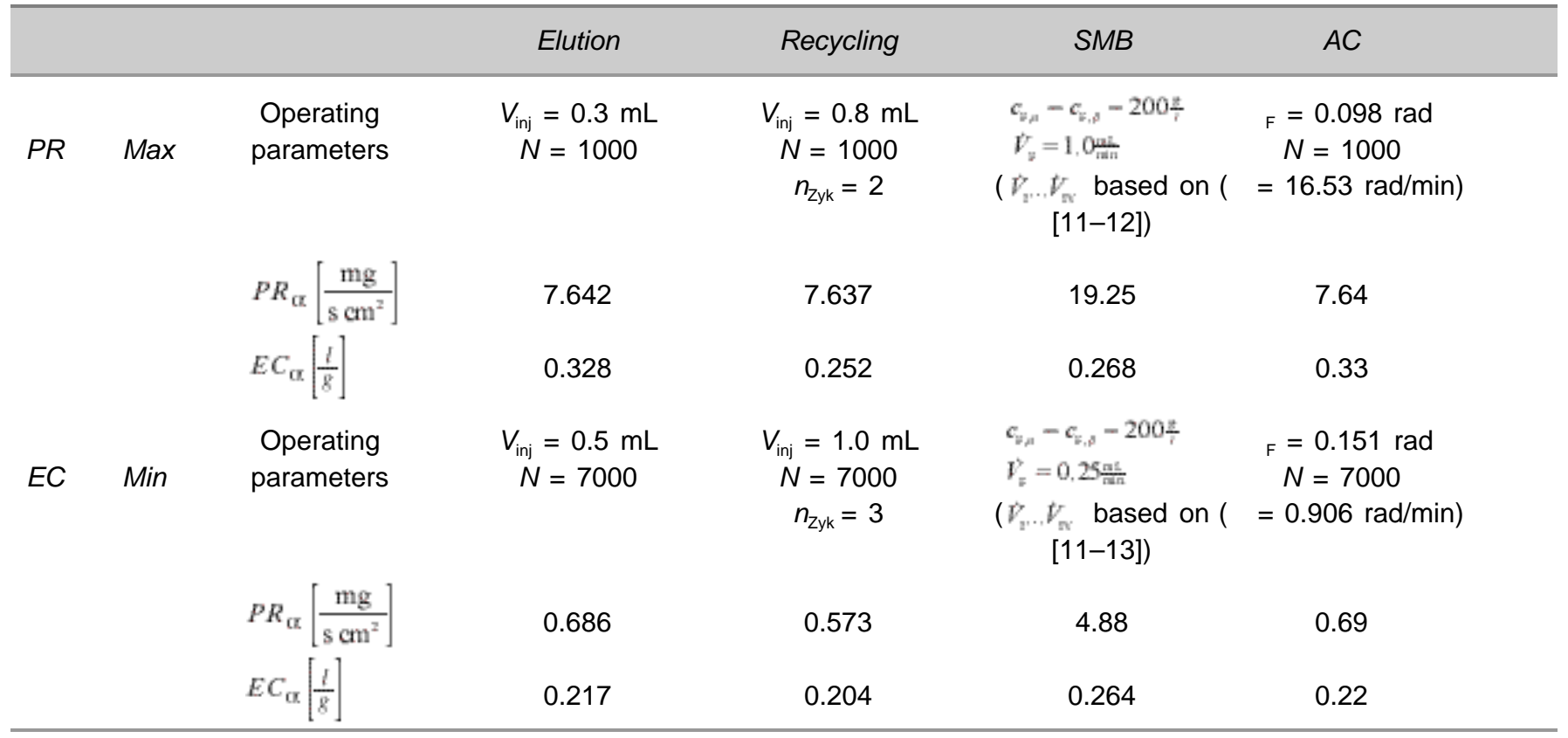

flowrates. Is the separation optimized with respect to a minimum eluent consumption $(E C \rightarrow$ Min) it is evident from table IV that there are very similar results for all four processes. However, compared with $P R \rightarrow$ Max the corresponding production rate is strongly reduced. In the parameter region covered, due to the assumed minimum value for the feed flowrate of $0.25 \mathrm{~mL} / \mathrm{min}$, the $\mathrm{SMB}$ process requires the largest eluent consumption. A further decrease of the volumetric feed flowrate would of course reduce the eluent consumption but would also cause a further reduction of the production rate.

From the obtained results it can be concluded that for the investigated separation of the two steroid isomers the maximization of the production rate is obviously the more useful objective function compared to the minimization of the eluent consumption. For the considered separation the SMB process appears to be the superior mode. However, a restricting remark should be finally added. In elution, recycling chromatography and annular chromatography different solvents can be applied as mobile phase and to dissolve the sample to be injected. This additional flexibility is not offered by the SMB process where in the standard mode only one solvent can be used. In the present case a feed concentration of $200 \mathrm{~g} / \mathrm{L}$ of the steroid isomers could be prepared only in dichloromethane and not in the $n$-hexaneMTBE mixture used as the mobile phase.

\section{Conclusions}

Four different chromatographic operating modes (elution, recycling, SMB and annular chromatography) were compared based on thermodynamic and kinetic parameters and an uniform model with respect to different performance criteria for the separation of a mixture of two steroid isomers.
The processes were designed to achieve either a maximum production rate or a minimum in the specific eluent consumption. In dependence on these objective functions for each process specific optimal operating parameters have been determined. The derived results and tendencies are valid just for the separation problem studied. A simple generalization is not possible due to the specific character of each problem. However, the mathematical model and the proposed methodology to compare different modes appear to be generally applicable.

\section{Acknowledgement}

The essential contributions of C. Heuer, H. Kniep and T. Falk are gratefully acknowledged. The author is further grateful for the financial support of Deutsche Forschungsgemeinschaft, Max-Buchner Forschungsstiftung, Schering AG and Dr. Ing. Herbert Knauer GmbH.

\section{References}

1. Unger, K. K. (Hrsg.) Packings and stationary phases in chromatographic techniques, Marcel Dekker, New York, 1989.

2. Guiochon, G.; Golshan-Shirazi, S.; Katti, A. Fundamentals of preparative and nonlinear chromatography, Academic Press, Boston 1994.

3. Ganetsos, G.; Barker, P. E. Preparative and production scale chromatography, New York, Marcel Dekker, 1993.

4. Heuer, C.; Hugo, P.; Seidel-Morgenstern, A. Chem. Eng. Sci. 1995, 50, 1115-1127.

5. Broughton, D. B. Sep. Sci. Technol. 1984, 19, 723-736.

6. Nicoud, R.-M. LC-GC Int. 1992, 5, 43-47.

7. Begovich, J. M.; Byers, C. H.; Sisson, W. G. Sep. Sci. Technol. 1983, 18, 1167-1177. 
8. Heuer, C.; Hugo, P.; Mann, G.; Seidel-Morgenstern, A. J. Chromatogr. A 1996, 752, 19-29.

9. van Deemter, J. J.; Zuiderweg, F. J.; Klinkenberg, A. Chem. Eng. Sci. 1956, 5, 271-289.

10. Wankat, P. C. AIChE J. 1977, 28(6), 859-867.

11. Rhee, H. -K.; Aris, R.; Amundson, N.R. First-order partial differential equations, Prentice-Hall, Englewood Cliffs, Vols. 1 and 2, 1986 and 1989.

12. Mazotti, M.; Storti, G.; Morbidelli, M. AIChE J. 1996, 42(10), 2784-2796.

13. Kniep, H. Ph. D. thesis, Universität Magdeburg, 1998.
14. Rouchon, P.; Schonauer, M.; Valentin, P.; Guiochon, G. Sep. Sci. Technol. 1987, 22, 1793-1833.

15. Heuer, C.; Kniep, H., Falk, T.; Seidel-Morgenstern, A. Chemie Ingenieur Technik 1997, 69, 1535-1546.

16. Nicoud, R. -M.; Seidel-Morgenstern, A. Isolation and Purification 1996, 2, 165-200.

17. Cremer, E.; Huber, H. Angew. Chemie 1961, 73, 461.

18. Felinger, A.; Guiochon, G. AIChE J. 1994, 40(4), 594-605.

19. Felinger, A.; Guiochon, G. J. Chromatogr. A 1996, 752, 3140. 